\title{
Realidade Virtual Imersiva para Reprodução Cinematográfica
}

\author{
Vitor Mendes Carvalho \\ Universidade Federal de Pernambuco \\ Recife, Brasil \\ vitor.mendescarvalho@ufpe.br
}

\author{
João Marcelo Xavier Natário Teixeira \\ Universidade Federal de Pernambuco \\ Recife, Brasil \\ jmxnt@cin.ufpe.br
}

Resumo-O projeto apresentado é um modelo protótipo de um sistema de vibração e ventilação que será utilizado como retorno háptico de vídeos escolhidos para reprodução. Este sistema foi projetado utilizando as linguagens Python3 e Arduino, juntamente com um microcontrolador e um circuito de servo motor. $O$ sistema criado será utilizado em um projeto semelhante ao Sensorama, uma máquina de reprodução de filmes que utiliza Realidade Virtual e possui vídeo estereoscópico, sensações de movimento, impacto, aromas, ventilação, som estéreo para simular a imersão no ambiente. Além disso, tem como objetivo ser uma máquina de simulação de experiências imersivas com finalidade educativa e de entretenimento.

Palavras-chave-Sensorama, Imersão, Retorno Háptico, Python3, Arduino.

Abstract-The presented project is a prototype model of a vibration and ventilation system that will be used as a haptic return for videos chosen for reproduction. This system was designed using Python3 and Arduino languages, together with a microcontroller and a servo motor circuit. The system created will be used in a project similar to Sensorama, a movie reproduction machine that uses Virtual Reality and has stereoscopic video, sensations of movement, impact, aromas, ventilation, stereo sound to simulate immersion in the environment. In addition, it aims to be a simulation machine for immersive experiences for educational and entertainment purposes.

Keywords-Sensorama, Immersion, Haptic Feedback, Python3, Arduino.

\section{INTRODUÇÃO}

A Realidade Virtual (RV) não é apenas uma novidade em um laboratório de pesquisa ou uma diversão em uma feira de ciências. Pouco mais de 50 anos após a introdução da televisão, a tecnologia de RV nos apresenta dispositivos como o head mounted display (HMD). O Oculus Rift, por exemplo, é um aparelho de televisor que se envolve em nossas cabeças, literal e metaforicamente [1], algumas vezes mostrando locais, objetos e até mesmo universos fantásticos de jogos, sendo apenas um observador, outras vezes utilizando nossos sentidos para causar sensação de inclusão no ambiente.
Os dispositivos digitais estão sendo cada vez mais adotados para fins de aprendizado e educação [2]. Pesquisadores têm explorado os benefícios e aplicações da realidade virtual (VR) em diferentes cenários e a RV possui muito potencial de aplicação na educação, despertando muito interesse de pesquisa nos últimos tempos [3]. Em virtude disso, desenvolver e demonstrar as utilidades da RV imersiva no meio acadêmico pode, além de proporcionar aprendizado na área, trazer benefícios educacionais e motivar os alunos a explorar suas funcionalidades e usabilidade.

A Realidade Imersiva (RI) é uma tecnologia a partir da qual é criado um ambiente virtual no qual os sentidos humanos são simulados, de modo que a interação entre o usuário e esse ambiente se aproxima de uma atividade no mundo real [4]. A primeira tentativa de criação de um aparelho para reprodução de filmes com uso de RI foi por volta dos anos 50, com a invenção do Sensorama de Morton Heilig, apontado como pai da Realidade Virtual no seu tempo [5], pois anos depois o reconhecimento no meio acadêmico iria Ivan Sutherland pela criação do HMD, afinal o projeto de heilig visava mais o empreendimento do que uma pesquisa. Morton propôs de forma mecânica uma engenhoca que reproduzia filmes 3D causando imersão sensorial do público através de física mecânica e óptica.

\section{A. O Sensorama}

Apresentado ao público em 1955 e patenteado em 1962, o Sensorama [6] foi uma máquina pioneira na RV imersiva. Seu criador, Morton Heilig, recebeu o título de pai da Realidade Virtual, ele era um criador de "multimídia" quando esse termo sequer havia sido inventado. Sua ideia foi criar um aparelho de RV imersiva que reproduzia filmagens de 2 minutos de duração, gravadas pelo próprio Heilig com o uso de uma câmera 3D, que foi construída utilizando um método estereoscópico de $35 \mathrm{~mm}$. 
Sua invenção simulava ao longo de sua reprodução a brisa no rosto, os ruídos do local, as vibrações dos impactos e o cheiro do ambiente, além de ter capacidade de atender quatro pessoas simultaneamente. Apesar de sua invenção ter sido considerada inovadora para seu tempo, Heilig nunca conseguiu financiamento para levar seu projeto para produção comercial.

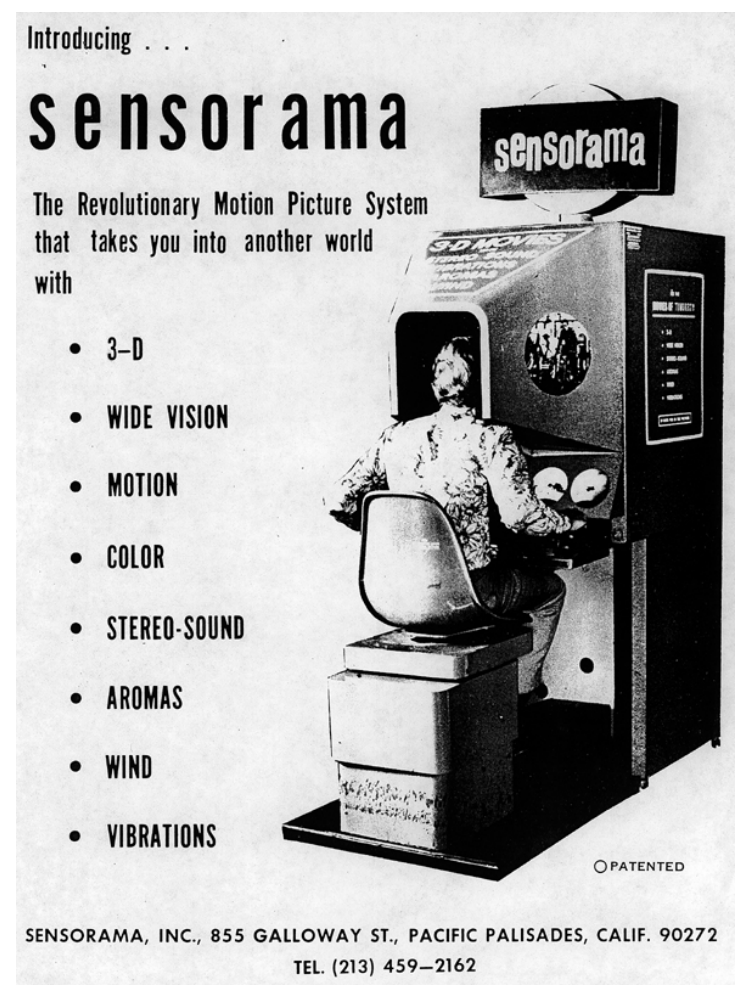

Figura 1. Poster de divulgação do Sensorama.

Mesmo que seu experimento não tenha se concretizado, sua invenção que tinha fins acadêmicos [6], chamou muita atenção na época. Por conta disso, a construção de um aparelho de RV imersiva com preço relativamente barato, utilizando eletrônica e programação para projetar, ao invés de ser completamente mecânico, facilitando a desenvoltura do projeto, pode vir a se tornar uma grande contribuição para desenvolvimento e estudo da RV nas universidades.

Neste projeto, objetivou-se desenvolver um aparelho de reprodução de vídeos com o uso de RV e Imersão sensorial com finalidade educacional e de entretenimento, com foco em proporcionar fácil acesso de diversas experiências práticas ao estudante, fazendo com que aumente o interesse tanto no que será apresentado no vídeo quanto no estudo da realidade virtual. E isso não vale apenas para crianças e adolescentes. Adultos, universitários, profissionais em cursos de capacitação, enfim, qualquer um pode tornar a aprendizagem mais interessante com a realidade imersiva, porém o alto preço que os dispositivos de realidade virtual custam hoje atrapalha a disseminação da tecnologia em instituições de ensino [4]. O objetivo final é a construção de uma máquina de imersão sensorial com a menor quantidade de hardware possível, com a garantia de que o usuário final do aparelho tenha a experiência de estar presente nos gravações estereoscópicas reproduzidas na máquina, tendo a possibilidade de sentir como seria estar realizando-as. Para a realização deste protótipo de Sensorama, foi implementada a funcionalidade de retorno háptico a partir do movimento de um vídeo.

\section{Desenvolvimento}

Como protótipo primário foi arquitetado um circuito de controle de um motor DC usando um Arduino UNO, que é uma placa baseada no microcontrolador Tmega328. Esse conjunto seria usado como retorno háptico de vibração para simular o impacto e movimento do vídeo reproduzido como mostrado na figura 4. Este mesmo modelo também pode ser aproveitado como ventilação se adicionada uma hélice ao motor.

Para controlar o comportamento do circuito foi desenvolvido um código em Python3 que reproduz o vídeo escolhido e paralelamente executa um processo de leitura de um arquivo de texto (de forma semelhante ao processo de leitura de um arquivo de legenda) que contém os comandos e seus determinados intervalos de tempo.

Os valores do arquivo de texto, como ilustrados na Figura 2, foram determinados de maneira arbitrária e manualmente com base no vídeo escolhido. Processo foi feito de maneira empírica e pode ser muito trabalhoso caso o vídeo seja muito longo.

Assim que a leitura de cada linha é concluída, os comandos são armazenados numa variável do tipo string onde os três primeiros valores correspondem ao tempo dos eventos, já os três últimos serão as instruções de regulação de velocidade de giro do motor.

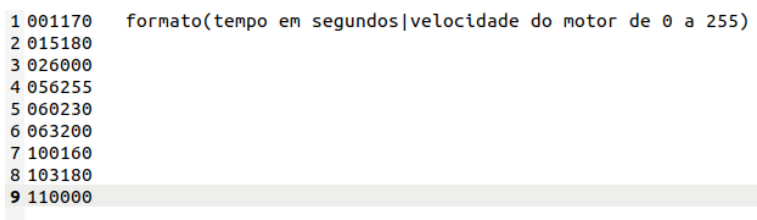

Figura 2. Arquivo de texto com comandos e tempo demarcados

Os dados são então separados e em seguida sofrem uma conversão para valores do tipo inteiro, como mostrado na Figura 3. Após isso, os primeiros são comparados com uma variável de contagem de tempo implementada em código para mapear o momento em que cada evento de troca de velocidade deve ocorrer. Quando os valores da variável de contagem de tempo forem iguais aos valores obtidos pela leitura do arquivo, será executado um comando de comunicação entre o código de Python3 e a porta Serial via Arduino IDE, onde as instruções de velocidade serão enviadas para o circuito motor.

Foi escolhido um vídeo estereoscópico com o objetivo de passar a impressão de 3D. Para a reprodução do mesmo 


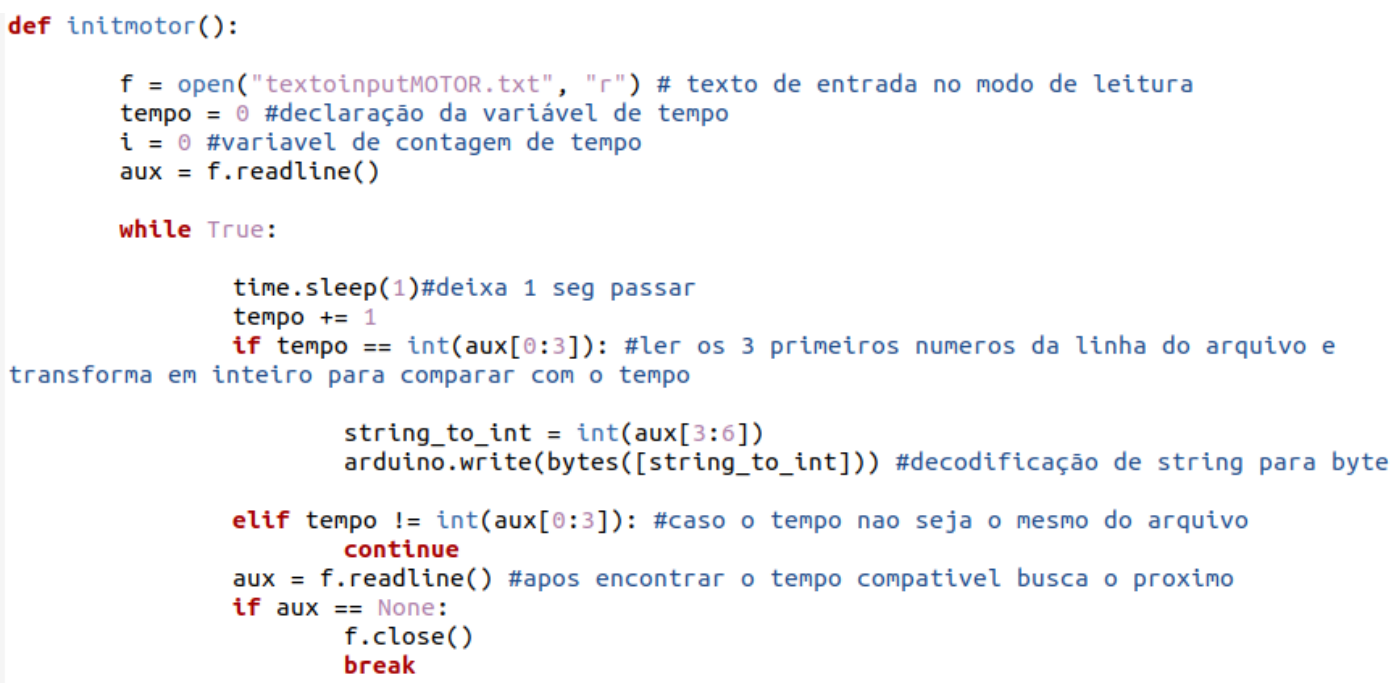

Figura 3. Função de Leitura e comunicação via Serial.

optou-se por utilizar o Pyglet ${ }^{1}$, uma biblioteca de Python3 útil para criação e reprodução de jogos e aplicações de multimídia, mas também poderia ser implementado via OpenCV ${ }^{2}$ dada a variedade de opções de tratamento de imagem oferecidas.

O paralelismo do código entre a reprodução do vídeo e a leitura e execução de comandos via porta Serial ocorreu com o uso da classe Process da biblioteca multiprocessing ${ }^{3}$ do Python3. O circuito do motor foi arquitetado usando um motor DC de $5 \mathrm{~V}$, uma protoboard, um diodo retificador modelo 1N4001, um transistor tipo NPN modelo PN2222, um Resistor de $270 \Omega$ (Ohms), jumpers e um Arduino Uno R3 com cabo de entrada USB, como ilustra a Figura 4.

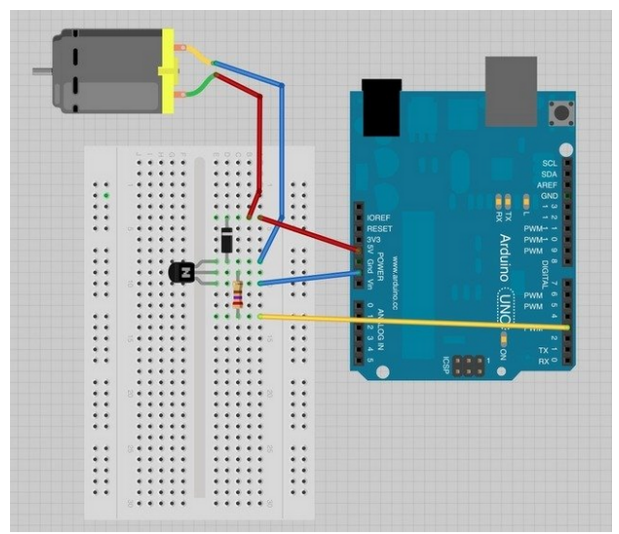

Figura 4. Circuito do motor DC.

O Objetivo final do projeto será construir um aparelho similar ao Sensorama, onde o protótipo apresentado faria parte de um sistema maior com controle de odores, ventilação

\footnotetext{
${ }^{1}$ http://pyglet.org/

${ }^{2}$ https://opencv.org/

${ }^{3}$ https://docs.python.org/2/library/multiprocessing.html
}

e movimento, além do vídeo estar devidamente adaptado para sensação 3D com saídas de som estéreo para causar sensação imersiva.

\section{RESUltAdOS}

Como resultado do protótipo primário obteve-se a sincronia da realização dos processos de reprodução do vídeo estereoscópico, de leitura e execução das instruções de velocidade do motor que foram definidas no arquivo de entrada. A alteração de velocidade gera a vibração esperada para causar sensação de movimento, além de poder ser usada como sensação de ventilação se adicionada uma hélice e realizada uma alteração no código para que possa reagir de forma diferente. $\mathrm{O}$ resultado de retorno háptico foi satisfatório dado o baixo custo do material utilizado, porém o processo de implementação de sensação de movimento deve ser manual para cada tipo de vídeo e se restringe a sensação de pequenos impactos e vibrações.

O sistema atual pode ser comparado com um protótipo do projeto HyCicle que envolve o uso de uma bicicleta montada em uma plataforma conectada a um controle computador. $\mathrm{O}$ computador monitora o pseudo-movimento da bicicleta e em resposta é capaz de gerar vários tipos de efeitos especiais [7]. Contudo o HyCicle possui um sistema háptico ativo em tempo real, diferente do sistema passivo proposto.

No vídeo ${ }^{4}$ do motor em funcionamento, o código é executado no terminal do Ubuntu e o motor realiza as transições de velocidade enquanto o vídeo é reproduzido. Até a conclusão do projeto final, é almejada a construção de um sistema de RI completo incluindo a sincronização do áudio estéreo por meio de código e liberação de aromas como um processo simultâneo a reprodução do vídeo. Além disso, planeja-se também a construção de um suporte físico para o sistema, que em homenagem ao Sensorama, terá um formato similar,

\footnotetext{
${ }^{4}$ https://youtu.be/kAlR_kCweG4
} 
porém será utilizada a tecnologia proposta no projeto em questão.

\section{CONCLUSÃO}

Neste projeto foi desenvolvido um sistema de retorno háptico para sensação de impacto e ventilação para um sistema de reprodução cinematográfica de RV imersiva, que para um protótipo inicial, mostrou resultados satisfatórios.

A curto prazo o sistema pode ser aproveitado para desenvolvimento de outras funcionalidades similares ao Sensorama, mas a longo prazo o MCU principal de controle deve ser alterado, realizando uma transição de Arduino Uno para um Raspberry PI, que possui compatibilidade com HDMI e é mais robusto que o microcontrolador atual, além de aumentar a portabilidade do sistema.

Somado a isso haverá a aquisição de novos servo motores para adaptar as funcionalidades mais complexas como a liberação de aroma. Pretende-se também definir uma especificação mais genérica para o arquivo que lista as ativações dos atuadores, de forma que seja possível suportar mais de dois dispositivos simultaneamente.

Além disso, pode ser necessário criar um método de construção automática do arquivo de texto com os comandos já definidos para facilitar a adaptação com qualquer tipo de novo vídeo que possa vir a ser adicionado futuramente à biblioteca de reprodução.

\section{REFERÊNCIAS}

[1] M. R. BIOCCA, Frank; LEVY, "Communication in the age of virtual reality." vol. 3rd. ed, p. 416, 1995

[2] C. ZAWACKI-RICHTER, Olaf; LATCHEM, "Computers \& education: Exploring four decades of research." Computers \& Education, vol. 122, pp. 136-152, 2018.

[3] J. Radianti, T. A. Majchrzak, J. Fromm, and I. Wohlgenannt, "A systematic review of immersive virtual reality applications for higher education: Design elements, lessons learned, and research agenda,' Computers \& Education, vol. 147, p. 103778, 2020.

[4] "Realidade imersiva: o que é, aplicações e tecnologias," FIA - Fundação Instituto de Administração, 2019.

[5] H. M. Hefner, "Morton heilig : Inventor vr," USC School of Cinematic Arts.

[6] M. HEILIG, "Sensorama simulator," Google Patents, 1961.

[7] P. BARKER, "Virtual reality: theoretical basis, practical applications," Research in Learning Technology, 1993. 